\title{
Information management for high content live cell imaging
} Daniel Jameson"1, David A Turner' ${ }^{2}$, John Ankers², Stephnie Kennedy², Sheila Ryan², Neil Swainston1, Tony Griffiths'3 ${ }^{3}$ David G Spiller², Stephen G Oliver ${ }^{4}$, Michael RH White ${ }^{2}$, Douglas B Kell ${ }^{1}$ and Norman W Paton*3

Address: ${ }^{1}$ Manchester Centre for Integrative Systems Biology, School of Chemistry, and Manchester Interdisciplinary Biocentre, University of Manchester, 131, Princess St, Manchester, M1 7DN, UK, 2Centre for Cell Imaging, School of Biological Sciences, Bioscience Research Building, Crown St., Liverpool, L69 7ZB, UK, ${ }^{3}$ School of Computer Science, Kilburn Building, The University of Manchester, Oxford Road, Manchester, M13 9PL, UK and ${ }^{4}$ Department of Biochemistry, University of Cambridge, Sanger Building, 80 Tennis Court Road, Cambridge, CB2 1GA, UK

Email: Daniel Jameson - daniel.jameson@manchester.ac.uk; David A Turner - D.A.Turner@liverpool.ac.uk;

John Ankers - J.M.Ankers@liverpool.ac.uk; Stephnie Kennedy - stephnie.kennedy@liverpool.ac.uk; Sheila Ryan - Sheila.Ryan@liverpool.ac.uk; Neil Swainston - neil.swainston@manchester.ac.uk; Tony Griffiths - tony@intetech.com; David G Spiller - spilldg@liverpool.ac.uk; Stephen G Oliver - steve.oliver@bioc.cam.ac.uk; Michael RH White - M.White@liverpool.ac.uk; Douglas B Kell - dbk@manchester.ac.uk; Norman W Paton* - norm@cs.man.ac.uk

* Corresponding author

Published: 21 July 2009

BMC Bioinformatics 2009, 10:226 doi:10.1186/147|-2105-10-226
Received: 9 February 2009

Accepted: 2I July 2009

This article is available from: http://www.biomedcentral.com/I47I-2/05/I0/226

(C) 2009 Jameson et al; licensee BioMed Central Ltd.

This is an Open Access article distributed under the terms of the Creative Commons Attribution License (http://creativecommons.org/licenses/by/2.0), which permits unrestricted use, distribution, and reproduction in any medium, provided the original work is properly cited.

\begin{abstract}
Background: High content live cell imaging experiments are able to track the cellular localisation of labelled proteins in multiple live cells over a time course. Experiments using high content live cell imaging will generate multiple large datasets that are often stored in an ad-hoc manner. This hinders identification of previously gathered data that may be relevant to current analyses. Whilst solutions exist for managing image data, they are primarily concerned with storage and retrieval of the images themselves and not the data derived from the images. There is therefore a requirement for an information management solution that facilitates the indexing of experimental metadata and results of high content live cell imaging experiments.

Results: We have designed and implemented a data model and information management solution for the data gathered through high content live cell imaging experiments. Many of the experiments to be stored measure the translocation of fluorescently labelled proteins from cytoplasm to nucleus in individual cells. The functionality of this database has been enhanced by the addition of an algorithm that automatically annotates results of these experiments with the timings of translocations and periods of any oscillatory translocations as they are uploaded to the repository. Testing has shown the algorithm to perform well with a variety of previously unseen data.

Conclusion: Our repository is a fully functional example of how high throughput imaging data may be effectively indexed and managed to address the requirements of end users. By implementing the automated analysis of experimental results, we have provided a clear impetus for individuals to ensure that their data forms part of that which is stored in the repository. Although focused on imaging, the solution provided is sufficiently generic to be applied to other functional proteomics and genomics experiments. The software is available from: fhttp://code.google.com/p/livecellim/
\end{abstract}




\section{Background Introduction}

Recent advances in biological experimental techniques have often been geared towards increasing throughput of data. In microscopy, the combination of fluorescently labelled proteins, combined with automated focus and image capture, has led to a large increase in "high content screening" of cells, for phenotype and protein localisation in response to a variety of environmental perturbations (e.g. [1-4]). The majority of these assays deal with cells at single time points, but more challenging, and potentially more useful for a systems biology approach involving mathematical modelling, is being able to track cellular functions in a number of individual live cells over a period of time.

We have been using high content cell imaging techniques

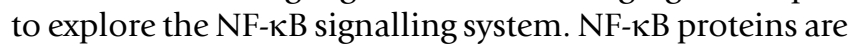
a family of transcription factors involved in the regulation of cell division, apoptosis and inflammation $[5,6]$. NF- $\kappa B$ is released from inhibitor kappa-B (IкB) in the cytosol and translocates into the nucleus where it activates transcription of target genes, including that of its inhibitor IкB. ІкB binds to NF- $\mathrm{KB}$ and transports the active complex back into a dormant cytoplasmic localisation [5,7]. The rela-

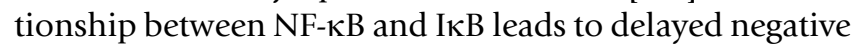
feedback, which generates oscillatory behaviour in NF-kB localisation [5]. These oscillations can be studied effectively using fluorescently tagged NF- $\kappa$ B and IкB proteins (Figure 1). However, the pathway is complex, and high throughput screening of samples is proving to be essential for investigation of the mechanisms that regulate these dynamic processes [5]. Additionally, an important part of our work concerns the construction of an accurate dynamic mathematical model of the NF-kB pathway which must be fitted and verified using experimental data.

Traditionally, experimental details and results are recorded in lab books. This enables an experimentalist to

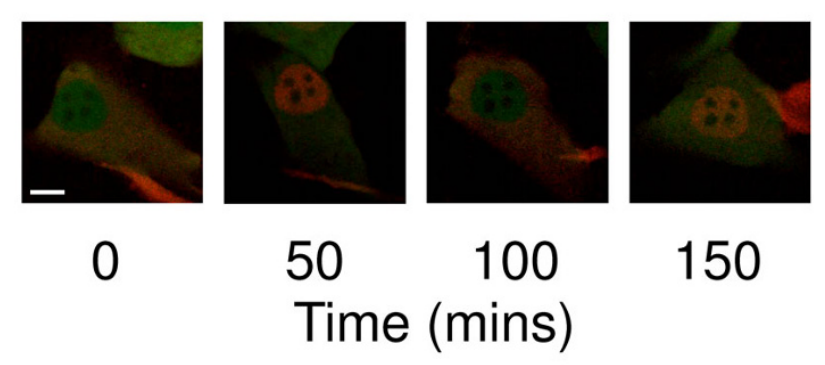

Figure I

Time course observing movement of fluorescently labelled protein involved in the NF- $\kappa B$ signalling system. Scale bar represents $10 \mu \mathrm{m}$. SK-N-AS cells transfected with $\mathrm{P} 53 \mathrm{dsredXP}$ and EGFP. quickly return to an experiment that they have performed; providing they can remember which book they recorded the information in. Whilst this may be sufficient for small self-contained experiments in small research groups, it does not, even at that scale, provide any facility to correlate seemingly disparate results and experiments between experimentalists unless links are drawn through discussions or by diligent project management. As the number of experiments performed and the number of measurements taken in each experiment increase, it becomes less likely that relevant associations will be identified, and more likely that important links will be overlooked, or at worst, experiments will be needlessly repeated.

Imaging experiments, such as those described, present additional problems, in that they generate large primary data sets with associated results that cannot be written into lab books. These may be stored on computer hard drives or DVDs, where typically only the experimentalist understands the precise system of nomenclature that they have used for their generated files, and experience suggests that even they may have some difficulty in discovering and interpreting datasets produced over an extended period.

In our specific environment, there is a requirement to archive in the region of 120 experiments per week, each one of which may be analysing up to 20 individual locations containing multiple cells over variable time courses. The image data are stored on RAID arrays providing a reasonable level of integrity for the data, but the amount of data generated presents a number of significant challenges for both information management and data analysis.

Systematic analysis of the time-series data gathered has been facilitated through the use of CellTracker $[8,9]$, which allows fluorescent intensity in the cytoplasm and nucleus of individual cells to be quantified and recorded automatically. This is performed by allowing the experimentalist to define nuclear and cytoplasmic boundaries for cells of interest, and then using a particle filter algorithm to track those boundaries as they move within the captured image field from frame to frame. The software measures the fluorescent intensities within each boundary for each captured frame, and then calculates the ratio between them.

However, the requirement for effective information storage and indexing has necessitated the design and implementation of an experimental metadata repository. A repository for high throughput imaging data in the context described above:

1. Enables associations to be identified between experimental details, results and subsequent analyses. 
2. Avoids repetition of experiments, as previous work is recorded.

3. Supports identification of relevant experiments; these may be from the same microscope, similar experimental conditions or have produced similar results.

\section{Requirements \& Contribution}

To address the problem outlined and provide the benefits described, requirements for the information management system were identified through an iterative series of meetings with both experimentalists and modellers. Initially these comprised of examining how data were manually gathered, recorded, and used, followed by discussions of what desirable attributes an information management system for these data would possess. The requirements identified were:

R1. Representation of experimental metadata and associated results.

\section{R2. Efficient mechanisms for data capture.}

R3. Efficient search mechanisms to identify experiments from metadata.

R4. Automated annotation of data derived from the images to allow identification of experiments whose results met certain criteria.

This paper presents an information management solution for high throughput cell imaging experiments, illustrated in the context of the NF- $\mathrm{KB}$ pathway, that addresses the above requirements by:

R1. Developing a new model that can capture both experiment descriptions and associated results, specifically descriptions of experimentalists, materials, protocols and microscope settings, as well as the output from the CellTracker software.

R2. Deploying the model using the Pedro data capture tool [10] to capture data that is then stored in a native XML database. The data capture tool utilises specially written plugins that allow the automatic import of microscope settings from microscope output files, as well as the re-use of previously described protocols and materials by importing them directly from the database.

R3. Providing a web-based search interface that uses canned (pre-defined) queries to retrieve details and results of experiments of interest. This provides the means to search over both experimental metadata and results.
R4. Computing summaries of the key features of time series image data (the change in fluorescence intensity of different cellular compartments over time and oscillations between these compartments) using a specifically developed algorithm, which are stored using the database from (R2) and accessed by way of the interface of (R3).

\section{Related Work}

Our requirements centre on the effective management of experimental metadata and results. Such solutions exist for local storage of other types of functional genomic experiments (e.g. maxd [11] for genomic expression data) and a variety of repositories cater for public storage and dissemination of experimental results (e.g. ArrayExpress [12], PRIDE [13]). Here we discuss available infrastructures for the management of microscopy image data, and note that at present no available solution provides for the local management of experimental metadata and results from high content imaging experiments.

Individual microscope manufacturers produce file formats specific to their own hardware and software (e.g. [14]). The associated software infrastructures typically do not provide complex search facilities across multiple experiments, and metadata associated with images is generally limited to details of imaging settings, omitting experimental context. In general the emphasis in vendor software is on experimental analysis rather than archiving and retrieval.

The Carl Zeiss LSM (Laser Scanning Microscope) format is a good example of a vendor microscope output. It is generated by Carl Zeiss confocal microscopes and the resulting files may be viewed with the LSM Image Browser [14]. The format consists of two parts, a Microsoft Access format MDB (Microsoft Access Database) file which describes microscope settings and references the image file for each location observed, and a series of .lsm (Laser Scanning Microscope) files that contain the imaging data for the observed locations. This records much of the data relevant to an imaging experiment, but does not record information relating to sample preparation and experimental design. Additionally, as there is a single image database for each experiment conducted, it is not straightforward to search across multiple experiments.

The Open Microscopy Environment (OME) is a fully featured repository for image data and associated metadata [15] designed to be utilised as a local archive. It is able to read image data from a wide variety of microscope formats, and is becoming ever more widely used. Once images are imported into the database, the user may annotate them using either pre-defined or custom tags. Additionally, external modules may be deployed that can interface with and use the data stored in OME, for exam- 
ple FindSpots [16]. OME is dependant upon the Bio-Formats library [17] for reading and writing to different microscopy image formats, and as such is limited in the metadata it can extract directly from the image files. For example, it is unable to extract the microscope settings from Zeiss LSM files. OME's emphasis is, however, on the management of images from microscopy experiments, rather than on the use of high throughput imaging as a functional genomics technique, as in this paper.

The Cell Centred Database (CCDB) is an online repository for managing and sharing image data [18]. Whilst originally geared to storing electron micrographs [19], it has been extended to encompass a wide range of microscopy techniques. All submitted data must be accompanied by project, experimental and protocol metadata. Along with original image data, CCDB stores analysis products derived from these, such as segmentations into substructures and reconstructions into three-dimensional objects. Submission to CCDB and subsequent annotation of images and input of instrument parameters is performed manually through a series of web-forms. CCDB is a solution for presenting and disseminating analysed datasets to a wider community, however recently it has started to be evolved to support work within an experimental environment, with the introduction of MyCCDB [18]. MyCCDB provides a personalised login allowing individuals or groups to upload data and assign privileges for others to view and manipulate the data without it being published on the public site.

The Centre for Bio-Image Informatics at Santa Barbara have produced Bisque (Bio-Image Semantic Query User Environment) [20]. It is centred around the storage of biological images and associated metadata. Personalised logins may be registered and individuals may upload images that may then be kept private or made public. Uploaded images may be directly annotated with drawing objects or else via associated meta-data tags. Images may also be analysed with a number of tools specifically tailored for analysis of retinal tissues (e.g. segmentation of layers, counting of nuclei) and microtubules (e.g. dynamics analysis and segmentation). Results of certain analyses are stored directly in the database, but there is no facility to search across them.

Over the last 15 years there has been a considerable amount of work on Content Based Image Retrieval (CBIR). CBIR aims to identify images or sequences of images based on the visual content of the images. This content is reduced to a set of features - perhaps colour, textures or shapes - that may then be compared to the features extracted from other images either by measuring some "distance" between them or applying some sort of probabilistic model to suggest how relevant an image may be [21]. The precise nature of the features extracted depends on the problem being addressed by the CBIR system.

CBIR has been applied experimentally to many fields of medical and biological imaging, e.g. asymmetry in brain MRI scans [22], features in X-rays [23], RNAi induced phenotypes of cells [24]. However, each system generated is optimized for a particular task and there are no generalised products available that are able to perform automatic feature extraction on the type of data that we gather other than CellTracker which we currently use.

CellTracker's feature extraction could be considered the first step in a CBIR system, however there is no requirement to identify specific images based on these extracted features, just a requirement to identify similar sets of extracted features. If a need were to be identified for identification of particular result sets based on aspects cellular morphology or another aspect of the captured images that are not explicitly extracted by CellTracker then some further form of CBIR may be an appropriate addition to our requirements list.

MyCCDB, OME and Bisque are strongly focused on image storage and retrieval, and address many of the problems which are faced by those who are generating large quantities of image data from disparate sources and those who need to make their image data available to a wider audience. Whilst our needs overlap with some of the features provided by these solutions, we differ in that there is no requirement to manage image data itself, but there is a specific requirement to record and manage the details of the context in which the images were acquired, and subsequently the numerical data derived from CellTracker analyses performed on them.

\section{Implementation \\ Data Requirements}

In addressing $\mathrm{R} 1$ it is important to understand the nature of the experiments undertaken, the data generated and the requirements for access. Figure 2 is a workflow diagram describes a generic microscopy experiment and data that is output from or required to describe each stage.

The requirement for the model is that it must represent the output from experiments and results along with descriptive metadata. The metadata should, in keeping with guidelines devised for other experimental metadata $[25,26]$, provide enough detail to validate the experimental methods used, minimise unnecessary repetition of experiments, and provide enough detail to repeat the experiment. There is no explicit requirement for the images to be archived along with the metadata, and as such it is reasonable to maintain a reference to the loca- 

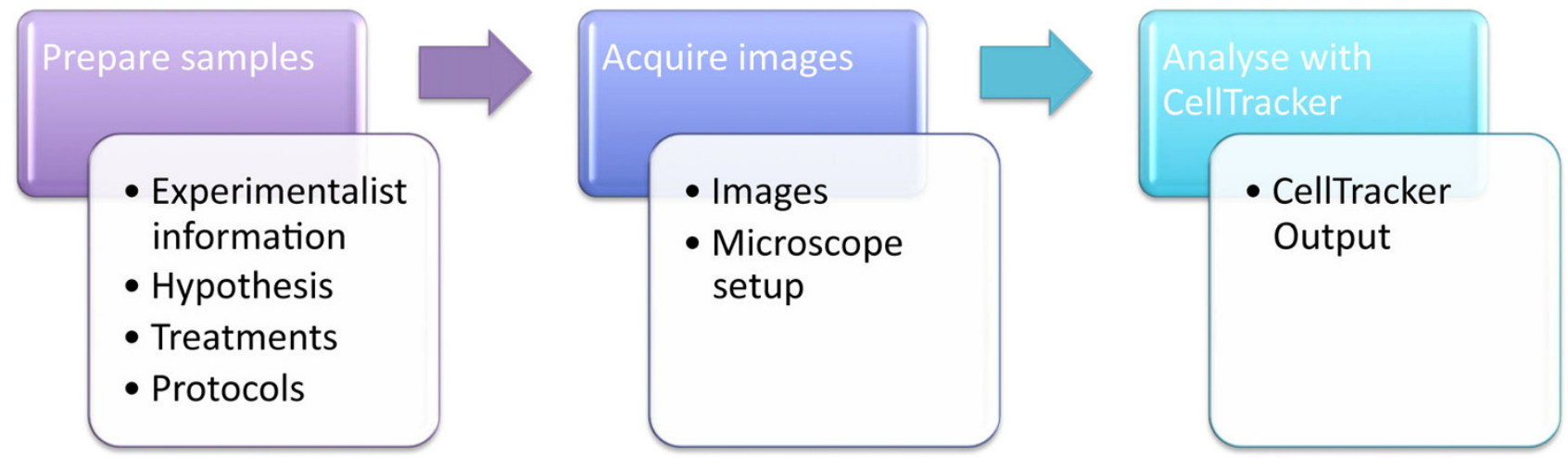

\section{Figure 2}

Workflow diagram illustrating the process of conducting a microscopy experiment. White boxes indicate the outputs from each stage that are required to describe the experiment.

tion of the image data, rather than storing the raw data in the database.

\section{The Model}

The microscopy experiments conform to the set-up illustrated in Figure 3. Cells are observed in multiple locations on a dish. Cells may be transfected with one or more plasmids and treated with one or more compounds. The plasmids and compounds may be identical at every location across the dish or, in the case of high throughput screenings, may vary by location. Each experiment performed may have more than one dish. Associated with the dish, or locations on the dish, are additional treatments and potential environmental perturbations. These in turn

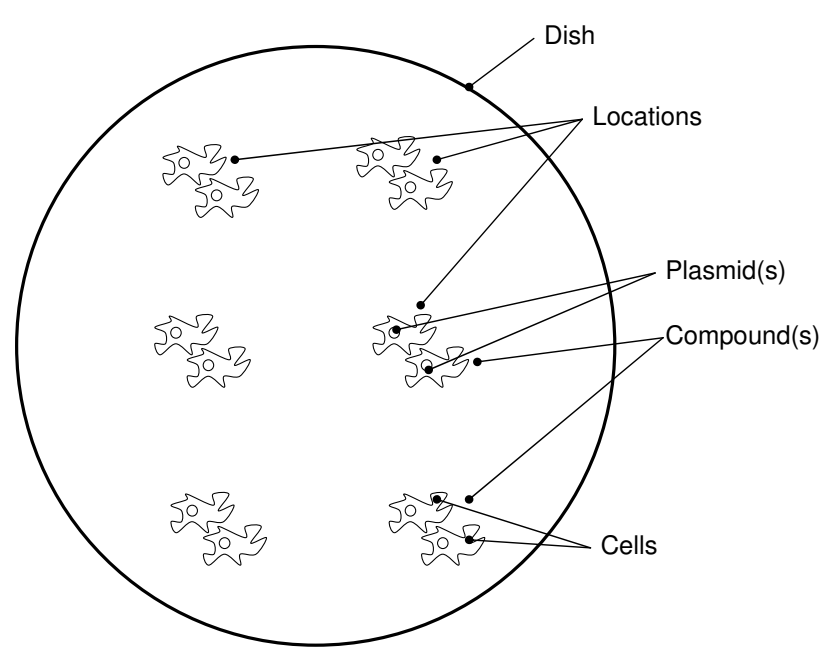

Figure 3

Elements that form an experimental dish unit. have protocols associated with them. This arrangement of entities forms the core of a model that can be used to describe an experiment and its associated results. Beyond that, on a more abstract level, is a description of the context within which it was created.

Figure 4 shows a UML diagram describing the relationships between the main objects that are associated with the Experiment class. Experiments are performed by people who belong to research groups. Experiments have a hypothesis, may be of various types (e.g., FISH (Fluorescence In Situ Hybridisation), FRET (Förster Resonance Energy Transfer) or Fluorescence), and may be performed using various techniques (e.g., confocal or wide field microscopy). Experiments can have many Dishes that were observed with a Microscope, as described above. The Experiment may be a spotted experiment, in which case cells treated in different ways may be applied to particular locations on the dish. Images are produced of Locations on the Dish, which once analysed, yield results that are directly related to that Location.

The model represents the physical and conceptual relationships between the elements that go to make up the experiment, but it must also capture the additional information provided by the microscope data files. The microscope records a large amount of information about its settings alongside the images it captures, such as laser intensities, filters, objectives, time points and tracks. This information is useful for validating procedures and replicating experimental configurations. Figure 5 shows the elements of the model that directly relate to the information provided in the Microscope data file and their relationship to the Dish, LocationReading and Results objects. 


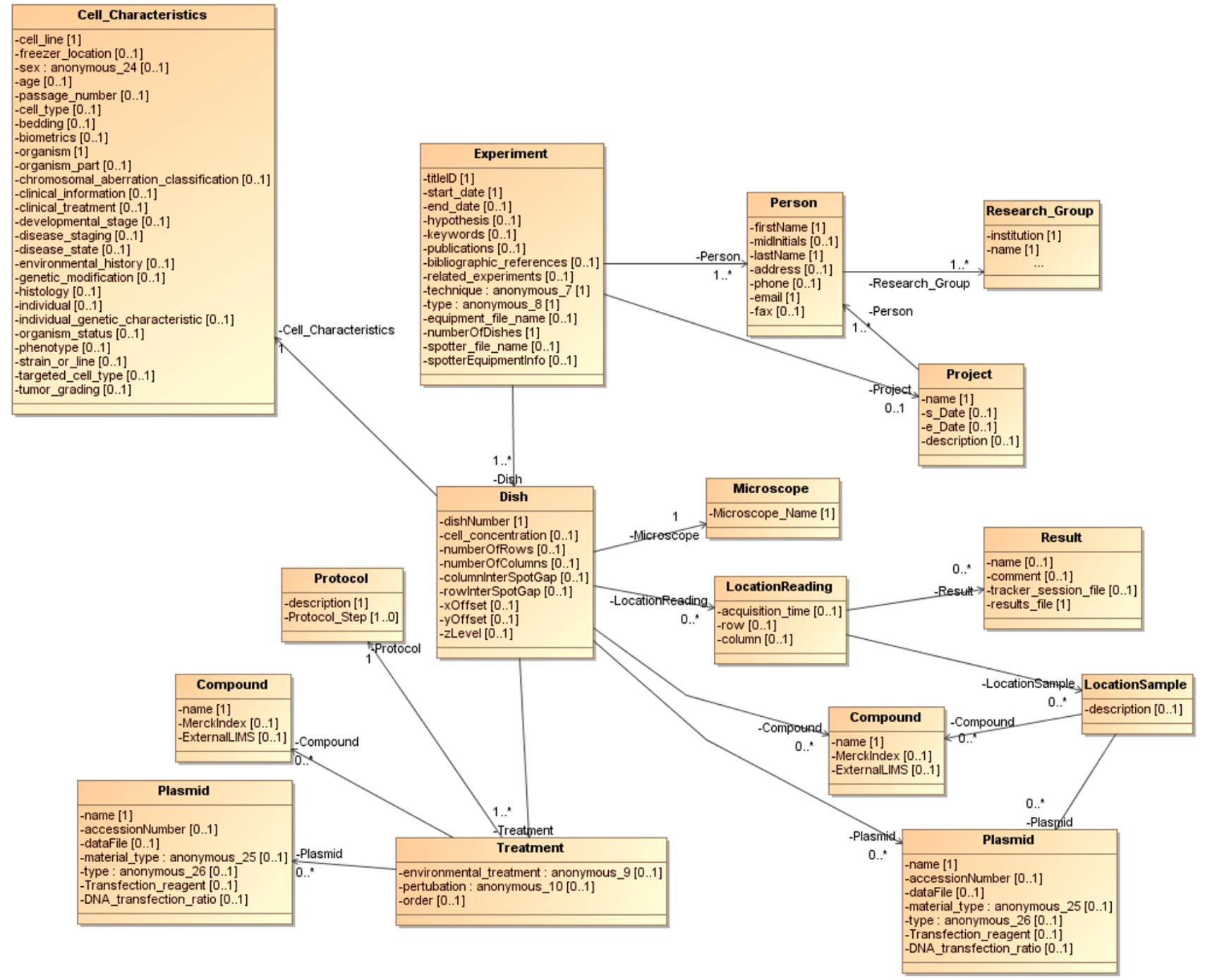

Figure 4

UML Diagram illustrating relationships between metadata elements and experimentally generated elements.

The remainder of the model is populated from CellTracker output and subsequent analysis performed on this. As CellTracker outputs XML (see additional files 1, 2, $3,4,5$ and 6), the model represents the CellTracker output format where a ResultTimeSeries has a sequence of ResultStates. CellTracker records the fluorescence in the nucleus and cytoplasm (CellularCompartments) of each Cell. Both Cells and CellularCompartments have fluorescence data captured about them (CellProperty) on one or more Channels. These Channels have a name that can be related back to the wavelength of light (and hence tagged protein of interest) which was being recorded on that channel, and intensity of the fluorescence recorded. Additionally, we use this results section of the model to hold information derived from an automated analysis of the CellTracker output (described in "Summarisation of Results" below).
For each recorded Result for a location on a dish, we generate AnalysisResults. The AnalysisResults consist of one or more AnalysedCells for that dish location, and for each of these we generate AnalysedChannels corresponding to the Channels that were recorded for that particular cell. The AnalysedChannels contain the details (Time, ratio value) of any Peaks relating to the movement of fluorescence between cytoplasm and nucleus. The content of this section of the model is shown in Figure 6.

\section{Implementation of the Model}

The data model is implemented as an XML Schema Definition (XSD), and thus the associated data are captured as $\mathrm{XML}$. This is advantageous for two reasons. Firstly, XML is a de-facto standard for the transfer of biological data. Secondly, we are able to make use of existing software infra- 


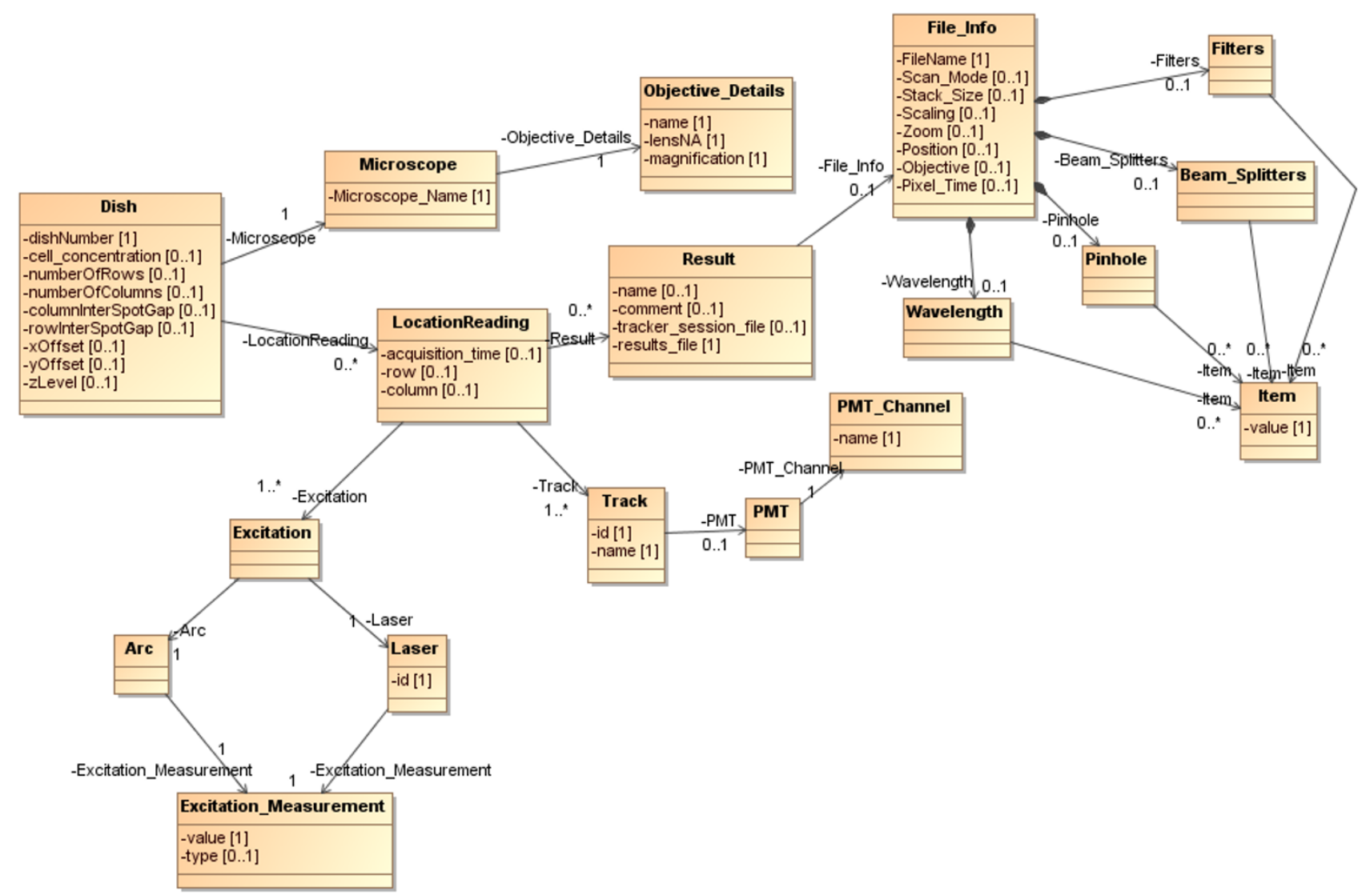

Figure 5

Classes derived from Microscope data files.

structure for capturing, managing and accessing XML data. The full XSD, along with example data files, are available in the additional files.

\section{Data Capture}

The data capture workflow is illustrated in Figure 7. The data are captured using Pedro [27]. Pedro is a flexible model-driven data capture tool that is used to populate XML documents that adhere to a predefined schema. The use of Pedro as a data capture tool for cell imaging data is discussed elsewhere [10]. To fulfil R2, data capture for the repository must capture the information specified by the model but minimise the amount of form-filling which must be performed by the experimentalist. This is achieved in two ways:

1. Making use of smaller repositories that store model fragments relating to commonly used items, such as Researchers, Protocols, Plasmids and Compounds. These can be selectively added to the main document being edited in Pedro.
2. Extracting metadata and experimental structure from the microscope generated data files and the CellTracker output files.

The extraction of metadata from the microscope data initially populates a Dish document element with the correct number of LocationReading elements. These in turn are automatically annotated with the correct image data file names and microscope settings.

For each LocationReading an analysis file is produced by CellTracker, which populates the result elements with the relevant time series data. After capturing the data, it may be saved as an XML document, or directly submitted to the database.

Once stored documents may be imported directly from the database back into Pedro for further editing and updating - results may be added or removed, 


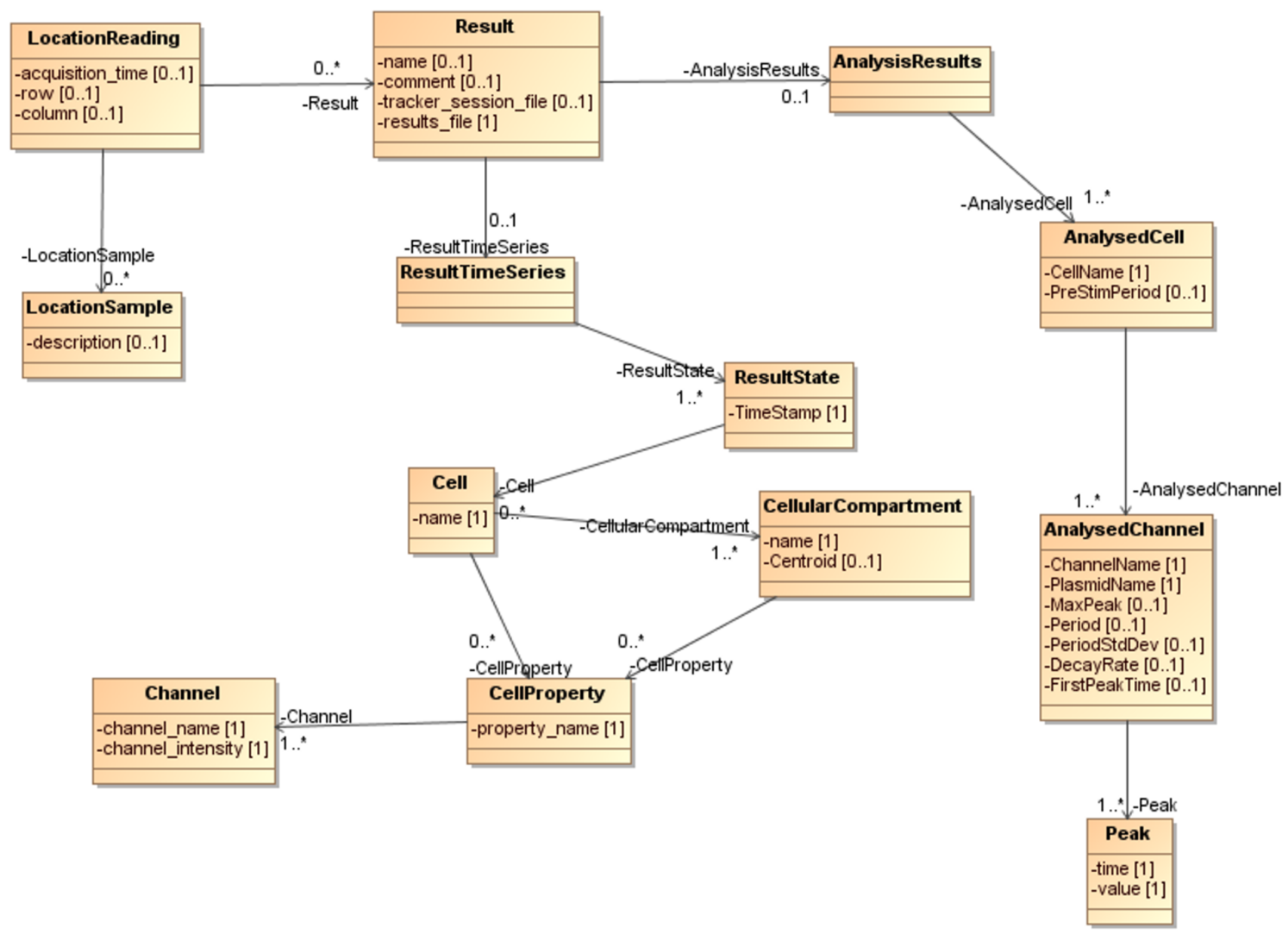

Figure 6

How the Result data structure relates to Location Reading.

\section{Data Storage}

The Tamino [28] or eXist [29] native XML DBMS can be used to implement the repository, which allows us to directly store the XML documents generated by Pedro during data capture. Additionally, as discussed below, the use of native XML storage provides for convenient generation of web pages using XSLT (XSL Transformation).

\section{Data Access}

Requirement 3 is for efficient searching over the archived metadata. Data needs to be accessed for two reasons, either updating, or searching and viewing. For updating, the data may be directly loaded back into Pedro from the database. For searching and browsing we have produced a web-accessible front end.

The front end is implemented as a series of Java Server Pages that send XQuery queries to the database and then transform the returned XML into HTML using XSLT documents.

After discussions with the experimentalists and modellers, initially ascertaining how data were currently consumed and subsequently examining what other questions may be asked of the data once stored in a database, the following requirements for querying the data were identified:

- List experiments by specific Experimentalist

- List experiments using a specific cell line

- List experiments performed on a specific date

- List experiments performed using a specific cell line and a specific plasmid 


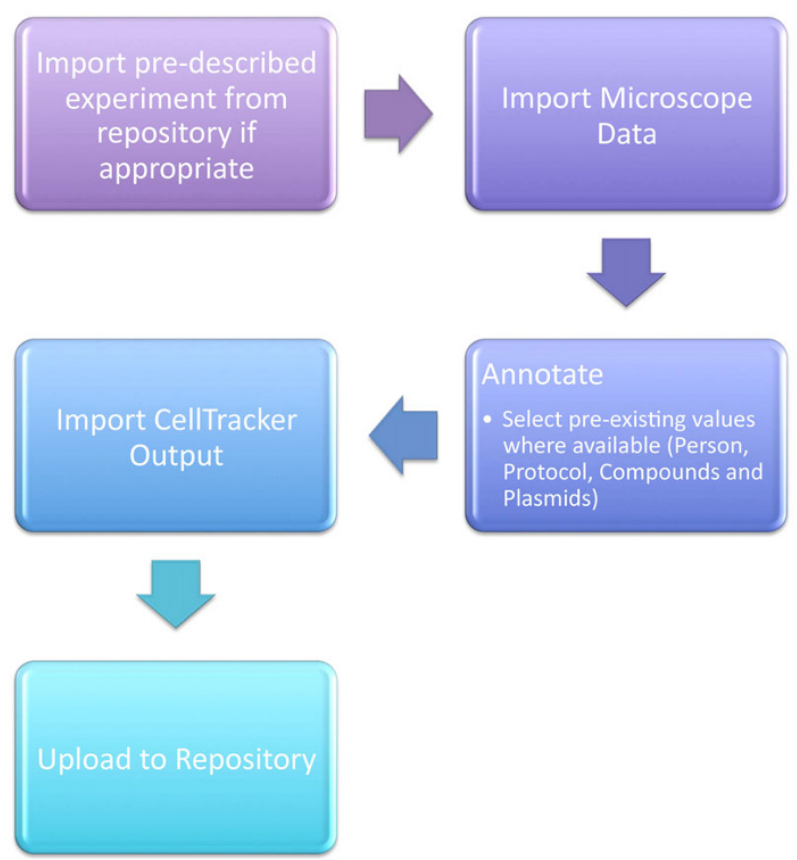

Figure 7

Workflow diagram illustrating the process of annotating and uploading an experiment to the database using Pedro.

- List experiments performed using a specific compound treatment

- List experiments performed using a specific compound and specific plasmid

- List experiments performed using two specific plasmids

As such, the emphasis of the search interface is on finding specific experiments rather than on more complex tasks such as comparison of experiments. As a result, the database is performing the role of an experimental catalogue.

Additionally, requirements were identified for retrieval of experiments, the results of which have certain characteristics:

- Show results where an oscillation of a specific period (give or take a certain amount) has taken place.

- Show results where a change in whole cell luminescence has taken place at a specific rate (give or take a certain amount).
All of these queries have been implemented, but as new requirements are identified, it is generally straightforward to add new queries.

The results of queries that return experimental lists are represented as a table of experiment titles. Clicking through provides a summary of the experiment and protocols used (Figure 8). From there it is possible to click through to the results of that experiment.

Queries interrogating the results yield a table of experiment titles, and selecting one takes the user directly to the results, highlighting any locations that fulfilled the query request. In order to allow querying over the experimental results we have implemented a summarisation algorithm that is run when CellTracker analyses are imported into Pedro.

\section{Summarisation of Results}

The facility to search the database for experiments whose results fit certain parameters, specified as R4, is important for modellers and experimentalists alike. We identified the following questions as being relevant for searching over experimental results:

Q1. Is there a movement of a measured fluorescent protein between the cytoplasm and nucleus, and if so when does this occur?

Q2. Are there subsequent movements resulting in an oscillation, and if so what is its period?

Q3. Is there a general trend in the overall level of measured fluorescence in a cell over time?

To meet these requirements, summaries are generated from the results of CellTracker analyses, which are stored in the database.

\section{Algorithm}

CellTracker generates a series of nuclear and cytoplasmic fluorescence intensities over time. By calculating and plotting the ratio between these intensities the translocation of a labelled protein from cytoplasm to nucleus may be observed as a peak (Figure 9). An algorithm has been developed to automate the detection of these peaks in the CellTracker output. This enables us to annotate the data as it is imported into the database with the times of NuclearCytoplasmic translocations and the period of any oscillation, which in turn allows us to answer questions Q1 and Q2. Q3 is addressed by calculating a regression line through the whole cell fluorescence over time.

The peak detection algorithm takes as its input nuclear and cytoplasmic fluorescence intensity values over time. It 


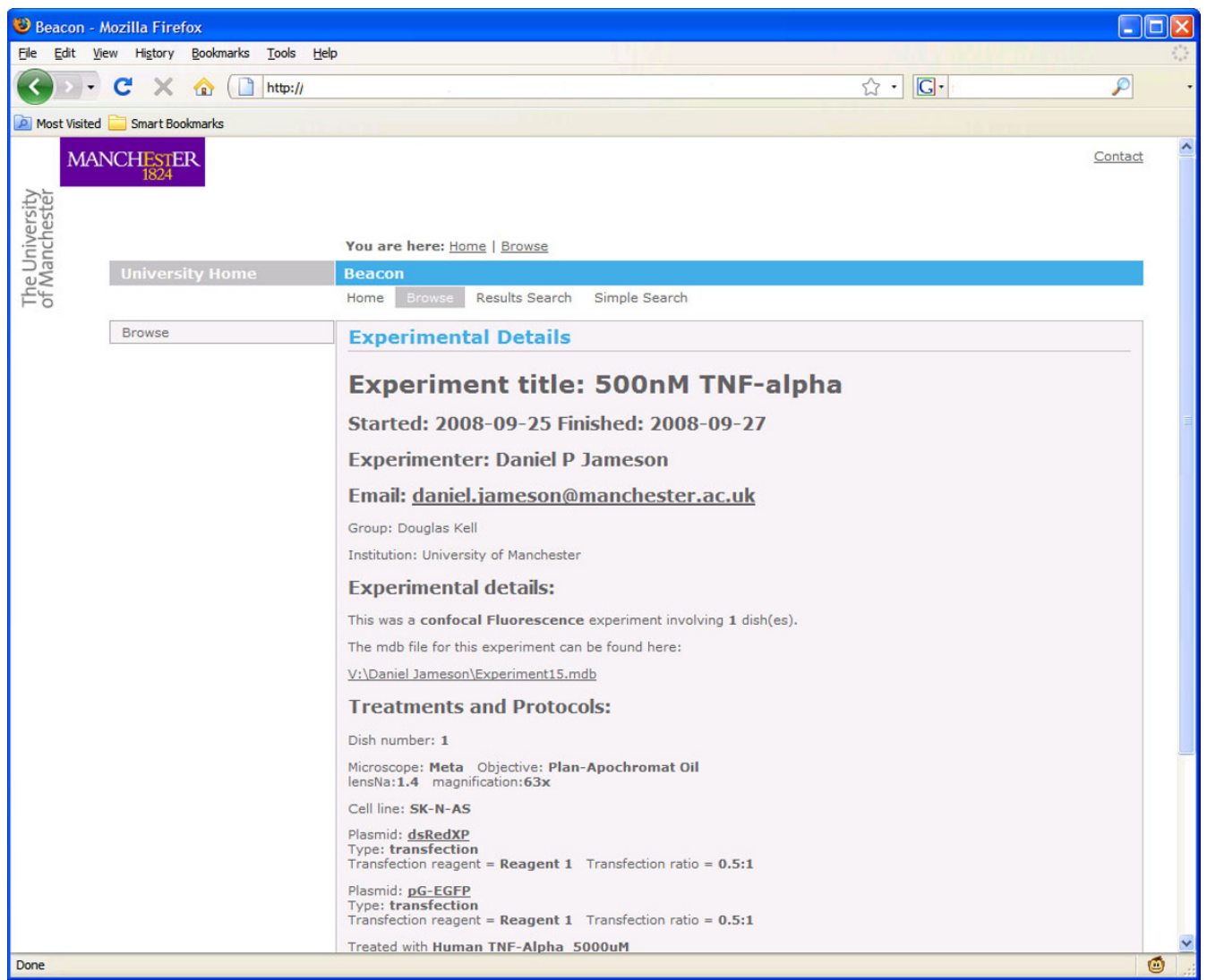

\section{Figure 8}

Experimental details from the metadata stored in the database. Canned search queries are in the list on the left hand side of the screen.

returns peaks identified by time and nuclear:cytoplasmic $(\mathrm{N}: \mathrm{C})$ ratio and the period of any identifiable oscillation. The algorithm is implemented in Java and based upon the Tom O'Haver's PeakFinder function for MatLab [30]. This was chosen as it had been specifically designed to identify positive peaks in noisy time-series data, and provided several parameters that could be adjusted to fit the data gathered from cell imaging.

The function accepts the data to be analysed along with parameters specifying the width of peaks to spot, a height threshold they must pass beyond (in our case this is a nuclear:cytoplasmic ratio), the width of the window to be used in the sliding average smoothing applied to the data, and a threshold gradient for the slope of the peak. The pseudocode for the algorithm is shown in Figure 10.

The algorithm has the following stages:

1. Detect prestimulation. Some experiments start when a chemical stimulus is added to the cells, others are run with a pre-stimulation period providing a base level for the N:C ratio. Adding the stimulus takes at least 30 seconds and hence an increase in the spacing of timepoints by at least this amount is indicative of a prestimulation period having been undertaken.

2. Calculate detection threshold. If a prestimulation has been undertaken, the detection threshold should be twice the standard deviation of the $\mathrm{N}: \mathrm{C}$ ratios in the prestimulation period (the criteria used by the experimentalists). Otherwise set the threshold to be 1.75 times the minimum recorded $\mathrm{N}: \mathrm{C}$ ratio - there is some tolerance with this value, however setting it much lower (1.5) or higher (2) increases the false positive and negative rates respectively.

3. Extrapolate values. If no prestimulation has occurred, the peak detection function is unlikely to identify the first peak, so a run-in of 10 timepoints with the N:C ratio at 0.1 below the starting value is prepended to the data. The peak detection function is also unlikely to a final peak that does not finish below the calculated detection threshold, hence if the final ratio val- 


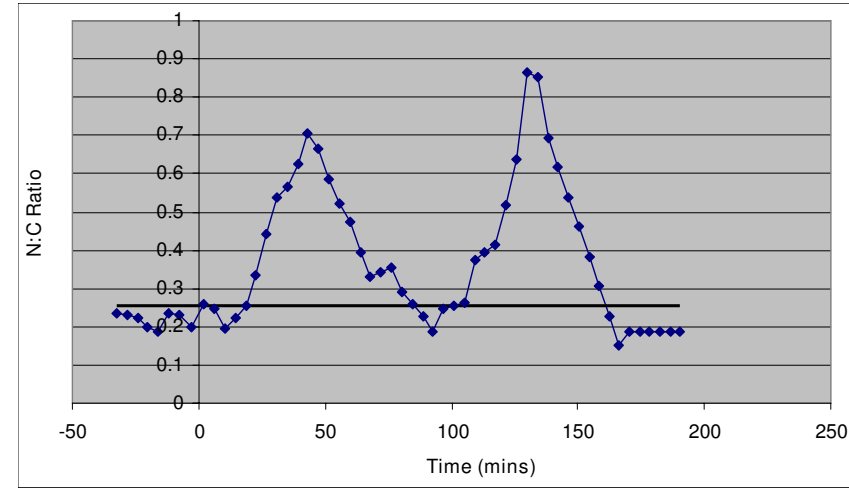

Figure 9

A peak in Nuclear:Cytoplasmic ratio of labelled protein, denoting a translocation between cellular compartments. Also indicated are calculated detection thresholds and data points extrapolated by the peak detection algorithm.

ues in the time series are a downward slope, the slope is extrapolated until it falls below the detection threshold. This is illustrated in Figure 7.

4. Optimise detection width. The detection width (that is the width of peak, in numbers of time points, to be detected) to be used is determined by repeatedly calling the peak detection function with increasing detection widths. When the optimal detection width is encountered, there is a jump in the detected location of the first peak (Figure 11).

5. Detection of peaks. Derivatives of the data are smoothed using an average sliding window of 10 time points in width (Lower values introduced extra false positives within available data, higher values increased false negatives). If a maxima in the data is encountered and the ratio value is above the detection threshold then a peak is recorded. If the detection width is less than 7 time points (as implemented in the original algorithm [30]), the location and height of that local maximum is recorded. For larger detection widths, a second order polynomial is fitted to the data and the location and height of its maximum is recorded.

\section{Results}

\section{Algorithm evaluation}

Data sets from two experimenters comprising noisy and smooth data (a subjective observation of how easily real peaks are visually identified, generally a product of the protein being observed and the amount of stimulation applied to the cell) from three different cell lines were used to parameterise the algorithm (Table 1, also see additional files 7 and 8). Experiments involved inducing the NF- $\kappa$ B pathway with TNF- $\alpha$ and then observing the local- isation of tagged proteins involved in that pathway. Three groups of unseen data were made available for testing the algorithm, which involved different experimenters and observed proteins (Table 2, also see additional files 7 and $9)$. The experimental protocol may be found in additional file 7 .

\section{Method of evaluation}

The algorithm analyses Nuclear:Cytoplasmic ratios from individual cells. As any subsequent analysis of protein movement is based upon the accurate detection of peaks in this data, the presence and absence of peaks in automatically and manually annotated results form a suitable metric by which to measure the effectiveness of the algorithm.

For each cell analysed a list of peaks detected by the algorithm is generated. This list comprises of the peak location (Time) and peak height ( $\mathrm{N}: \mathrm{C}$ ratio). These are then scored against manually observed peaks in the same data. The possible outcomes are:

- True positive (TP): the peak is detected by the algorithm and also observed as significant.

- False positive (FP): the peak is detected by the algorithm but not observed as significant.

- False negative (FN): no peak is detected by the algorithm but one is observed as significant.

Observations are subjective, in that they are performed by individuals who may have a preconceived idea of what their results should look like and also dependant on the particular parameters of an experiment. For example, depending on the treatment applied to them, some cell lines generally do not show any oscillations of the protein being observed between cytoplasm and nucleus, and hence an observer may be less likely to identify a small secondary peak as real.

The expected results from the test data sets were known in advance; the algorithm results for the test data were scored by the experimentalists.

\section{Results}

The effectiveness of the algorithm is illustrated by Recall $(\mathrm{TP} /(\mathrm{TP}+\mathrm{FN}))$ and Precision $(\mathrm{TP} /(\mathrm{TP}+\mathrm{FP}))$. TP, FP and FN are True Positives, False Positives and False Negatives respectively. A precision score of 1.00 would mean that every peak identified was a true peak, but would not give an indication of how many true peaks were missed. A recall score of 1.00 would mean that all the peaks present in the data were correctly identified as peaks, but would not give an indication of how many non-peaks were iden- 


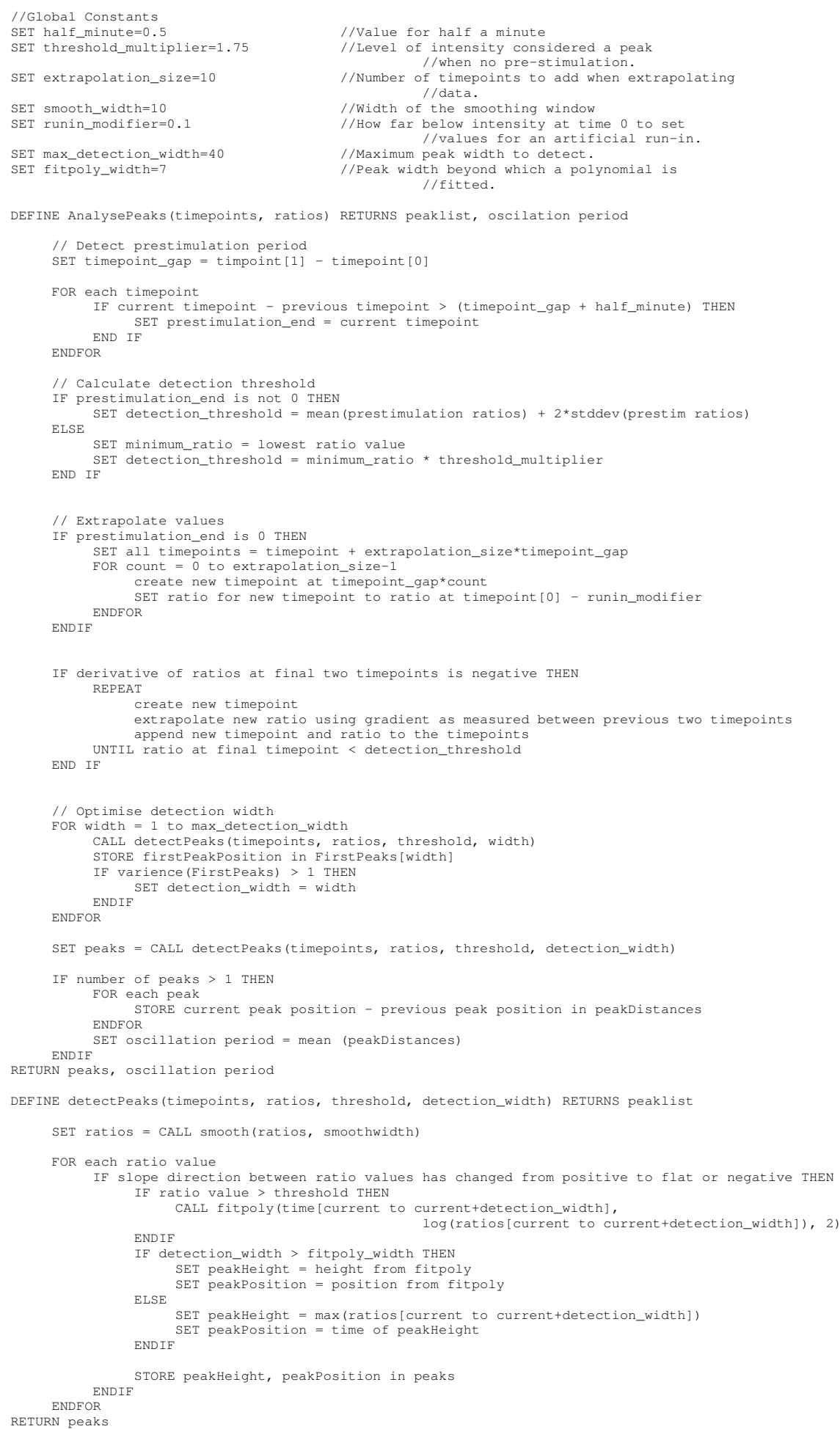

Figure 10

Pseudocode for the peak detection algorithm. fitpoly is a Java implementation of the MatLab Polyfit function which finds the coefficients of a polynomial which fits the specified data - fitpoly $(x, y, n)$ where $x$ and $y$ are vectors of the $x$ and $\mathrm{y}$ values and $\mathrm{n}$ is the order of the polynomial to be fitted. 


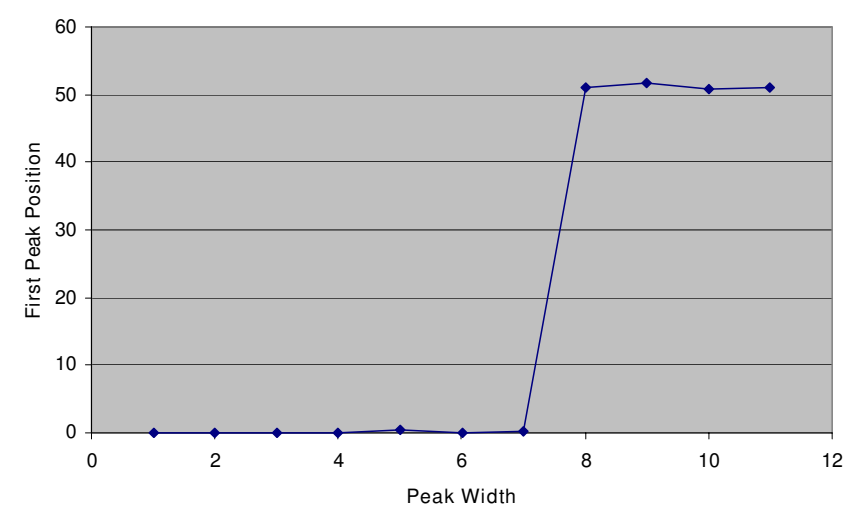

Figure I I

Detected position of first peak with increasing peak detection width.

tified as peaks. Taken together, these metrics give a good indication of the effectiveness of the algorithm. The training results (Table 3 and additional file 10) show an overall precision of 0.84 and a recall of 0.93 . Results from test data, representative of the data that is stored in the database, are shown in Table 4 (also see additional file 10). Here the overall precision is shown to be 0.71 and recall is 0.83 .

\section{Discussion of Algorithm Performance}

With the training data, the algorithm performs well, successfully identifying over $90 \%$ of peaks in the data presented to it with a precision of 0.84 . These were the data used to optimise the parameters of the algorithm and our approach to handling the data; as such, we expect good performance. This result also shows that our optimization has not been so strict as to yield a $100 \%$ score on the training data. Such a situation would be undesirable, indicating that the algorithm had little capacity to generalize beyond the training data. The true indicator of performance is through the analysis of unseen test data. With the test data the performance is less robust (identifying $83 \%$ of peaks with a precision of 0.71 ). However, almost half of the test data $(45 \%)$ were rated as noisy by the experimentalists as opposed to $16 \%$ in the training data.

The automatically generated data summaries aim to facilitate the identification of experiments in the database that may have yielded results of interest. Although we do not successfully identify every peak in the data, given that experiments invariably measure multiple cells in multiple locations, we can expect that a search over the results (of the nature Q1, Q2 or Q3) will return an experiment of interest unless the numbers of cells showing nuclear-cytoplasmic translocations are low. Figure 12 shows the results of a sample search over stored data for experiments where an oscillation was observed with a period of 100 $(+/-5)$ minutes.

Whilst the peak detection threshold and smoothing width values have performed well with the peaks generated by protein translocations within our experimental system, it is likely that they would require adjustment for data gathered in different circumstances. Further analysis of the data we are acquiring may lead us to be able to define features within the data (such as that described in Figure 9) that will allow us to dynamically assign these parameter values whilst the algorithm is running. Future development of the algorithm and associated infrastructure will allow stored data to be re-analysed using different parameters.

\section{Discussion}

The success of the work can be measured by how well it meets the requirements:

Table I: Training data used.

\begin{tabular}{lllllll}
\hline Data Set & Experimenter & Cell Line & Data type & Pre-stimulation for baseline? & Cell Count & Tracks \\
\hline TI & I & SK-N-AS & Noisy & Yes & 16 & 16 \\
\hline T2 & I & SK-N-AS & Noisy & Yes & 26 & 22 \\
\hline T3 & I & SK-N-AS & Smooth & Yes & 26 & 26 \\
\hline T4 & I & SK-N-AS & Smooth & Yes & 25 & 32 \\
\hline T5 & 2 & SK-N-AS & Intermediate & No & 26 \\
\hline T6 & 2 & HeLa & Intermediate & No & 51 & 97 \\
\hline T7 & 2 & MEF & Intermediate & No & 26 \\
\hline
\end{tabular}

Where Tracks is larger than Number of Cells, multiple tagged proteins were being monitored in each cell. 
Table 2: Test data used.

\begin{tabular}{|c|c|c|c|c|c|c|}
\hline Data Set & Experimenter & Cell Line & Data type & Pre-stimulation for baseline? & Cell Count & Tracks \\
\hline 1 & I & SK-N-AS & Intermediate & Yes & 21 & 21 \\
\hline 2 & I & SK-N-AS & Smooth & Yes & 21 & 21 \\
\hline 3 & 1 & SK-N-AS & Noisy & Yes & 23 & 23 \\
\hline 4 & 3 & HeLa & Noisy & No & 10 & 20 \\
\hline 5 & 3 & HeLa & Noisy & No & 12 & 24 \\
\hline 6 & 3 & SK-N-AS & Noisy & No & 12 & 24 \\
\hline 7 & 3 & SK-N-AS & Noisy & No & 12 & 24 \\
\hline 8 & 4 & HeLa & Intermediate & No & 102 & 102 \\
\hline
\end{tabular}

R1. Logical and appropriate representation of experimental metadata and associated results. The data model is general enough to describe a variety of microscopy experiments (fluorescence, luminescence, FISH and FRET) in both high and low content screening configurations, but it is specific enough to ensure effective validation of captured data. The model also represents the results generated by CellTracker. This may be contrasted with other data models for microscopy data (e.g. [15]) which focus on the context of an image rather than the details of the experiment and its result.

R2. Efficient mechanisms for data capture. Pedro has proved to be an adaptable and efficient data capture tool. By extracting details of microscope settings, locations observed under the microscope and data channels recorded from files generated by the equipment, and analysis results from the output of CellTracker, the

Table 3: Training data algorithm effectiveness.

\begin{tabular}{lll}
\hline Data Set & Recall (TPR) & Precision \\
\hline TI & 1.00 & 0.58 \\
\hline T2 & 0.89 & 0.84 \\
\hline T3 & 0.93 & 0.76 \\
\hline T4 & 0.94 & 0.97 \\
\hline T5 & 1.00 & 0.58 \\
\hline T6 & 0.89 & 0.93 \\
\hline T7 & 0.94 & 0.86 \\
\hline Overall: & $\mathbf{0 . 9 3}$ & $\mathbf{0 . 8 4}$ \\
\hline
\end{tabular}

TPR is True Positive Rate - TP/(TP+FN). Precision is TP/(TP+FP). time taken to submit a fully annotated experiment to the repository is roughly $10-15$ minutes. This is very rapid when compared to the length of time it can take to annotate other functional genomics type experiments (e.g., microarrays [31]) using standard tools.

R3. Efficient search mechanisms to identify experiments from metadata. The model represents both the metadata and acquired/analysed data for each experiment over which XQuery is used to implement canned queries that return results as XML documents. These documents are then formatted into appropriate reports using XSLT. As the reports are generally of similar format, implementing new queries is simply a matter of writing a new XQuery to retrieve data meeting the desired parameters.

Table 4: Test data, algorithm effectiveness.

\begin{tabular}{ccc}
\hline Data Set & Recall (TPR) & Precision \\
\hline I & 0.80 & 0.85 \\
\hline 2 & 0.85 & 0.90 \\
\hline 3 & 0.84 & 0.89 \\
\hline 4 & 0.73 & 0.29 \\
\hline 5 & 0.77 & 0.40 \\
\hline 7 & 0.44 & 0.17 \\
\hline 8 & 1.00 & 0.54 \\
\hline Overall & 0.87 & 0.87 \\
\hline
\end{tabular}




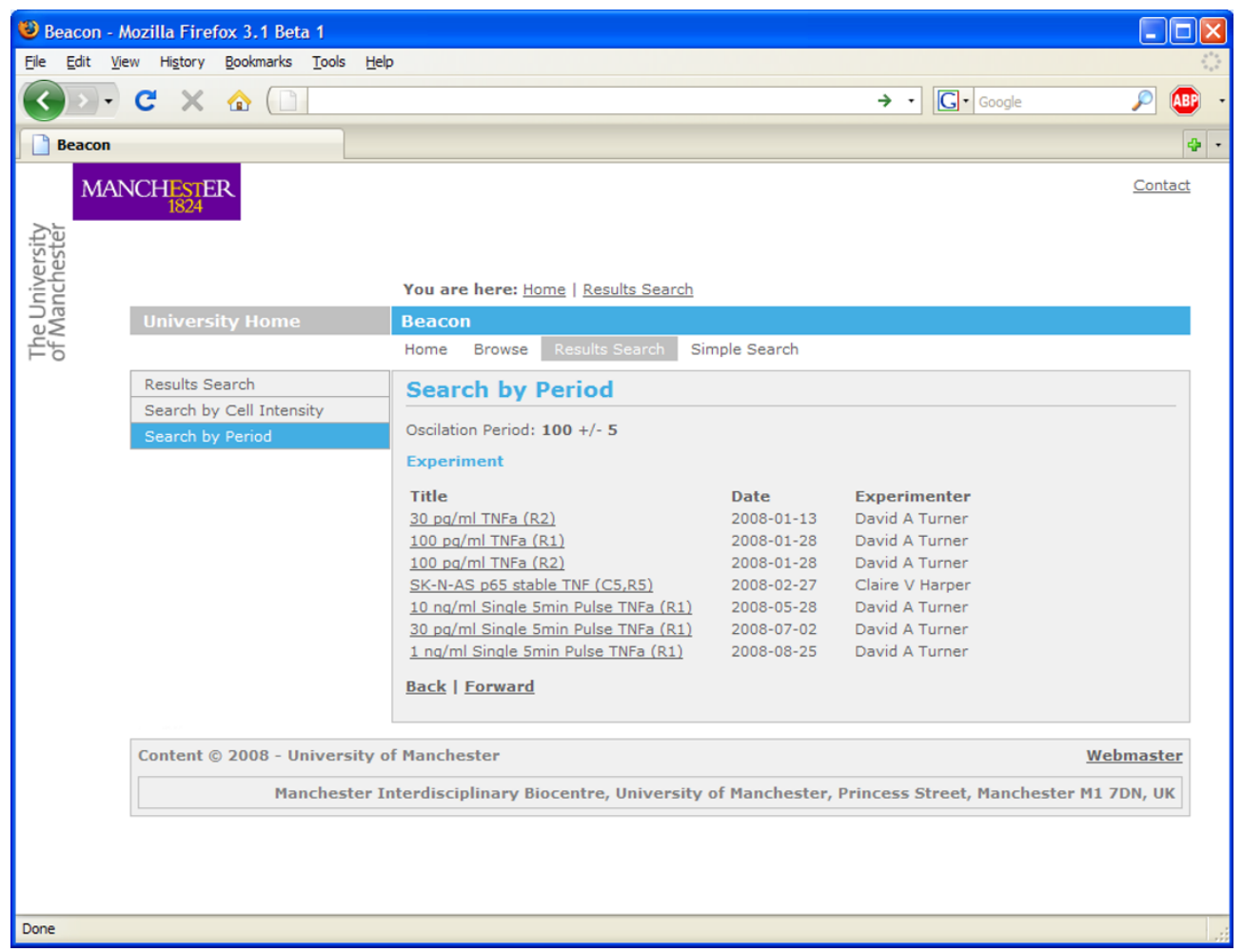

Figure 12

Searching stored experiments using a specific period of oscillation.

R4. Automated annotation of results to allow searching by result. This has been successfully implemented and performs well with currently available data. As this feature facilitates the identification of features within data it also adds impetus for experimentalists to submit their data to the database.

The effective modelling and storage of high throughput imaging data will remain an issue as technology improves and throughput rates increase. Our repository is a fully functional example of how these data may be effectively indexed and managed to address the requirements of end users. Particularly we move away from simply providing an index of contextual information about experiments and allow this to be complemented by indexing and describing the content of data gathered in these different contexts.

The system is currently in use by a group of 10 users who are all based in the same location. However, the repository is currently housed on a central server that is accessible from any authorised (this is dependant on server configuration) computer on the internet. The client Pedro software for submitting data to the database may also be run from any machine connected to the internet. This wide accessibility enables all individuals within a group to have their own client on their own machine for data submission without having to rely on access to a specific machine and would also lend itself to situations where collaborations require individuals from multiple institutions to have access to the repository.

The novelty of our system, and a key factor in its uptake by experimentalists, has been the inclusion of the peak spotting algorithm enabling the correlation of results across disparate experiments. With many biological repositories, data entry can be time consuming and, understandably, many individuals are disinclined to partake in this activity. By adding extra value to what would otherwise be simply a metadata and results repository, there is a clear benefit for individuals to ensure that their data forms part of the searchable corpus.

Whilst other solutions currently available address the management and analysis of image data, the database presented here demonstrates a solution and model for the effective management of experimental data gathered from high content live cell imaging. Despite the system being tailored to a specific arrangement and set of techniques, the approach and model are generic enough to be applicable to other functional proteomics/genomics experimental set ups. 


\section{Availability and Requirements}

- Project name: livecellim

- Project home page: http://code.google.com/p/live cellim/

- Operating system(s): Windows XP, Mac OS X 1.5.4 or later.

\section{- Programming language: Java}

- Other requirements: Minimum 2 GB RAM. JRE 6.0, Apache Tomcat 6.0, eXist-DB 1.2.4 or later.

- License: BSD

\section{Authors' contributions}

DJ wrote the text, refined the data model, refined the data capture process and tools, and designed, implemented and tested the peak spotting algorithm. TG designed and implemented the first version of the database and data capture tools. NS wrote the servlet architecture that is used by the front end along with many utility libraries. DAT, JA, SK, and SR provided and analysed test data for the algorithm as well as contributing through many discussions to the final data model. DS and MRHW supervised the microscopy experiments. SGO, DBK and MRHW supervised the application-oriented aspects of the project, and NWP supervised the computational aspects. All authors read and approved the final manuscript.

\section{Additional material}

\section{Additional file 1}

Installation instructions. How to install a working instance of the image database.

Click here for file

[http://www.biomedcentral.com/content/supplementary/1471-

2105-10-226-S1.rtf]

\section{Additional file 2}

Data model XML schema. This XSD document describes the data model used to capture the data relating to imaging experiments for the database. Click here for file

[http://www.biomedcentral.com/content/supplementary/1471-

2105-10-226-S2.zip]

\section{Additional file 3}

Sample experiment XML files. This file contains both a minimal experimental annotation and a complete annotation which includes results in $X M L$ format that may be uploaded to the database. The complete.xml file in the zip archive also contains CellTracker output XML within the $<$ Result $>$ tags.

Click here for file

[http://www.biomedcentral.com/content/supplementary/14712105-10-226-S3.zip]

\section{Additional file 4}

Database web front end application. The web application front end for deployment on a Tomcat server.

Click here for file

[http://www.biomedcentral.com/content/supplementary/1471-

2105-10-226-S4.zip]

\section{Additional file 5}

Pre-configured Pedro data capture tool. Pedro data capture tool configured to function with eXist XML database.

Click here for file

[http://www.biomedcentral.com/content/supplementary/1471-

2105-10-226-S5.zip]

\section{Additional file 6}

Source code. Java source code for the Pedro plugins and front end elements.

Click here for file

[http://www.biomedcentral.com/content/supplementary/14712105-10-226-S6.zip]

\section{Additional file 7}

Test and Training Data Details. Details of protocol, cell lines and plasmids for the Test and Training Data.

Click here for file

[http://www.biomedcentral.com/content/supplementary/14712105-10-226-S7.xls]

\section{Additional file 8}

Training Data. Spreadsheet files containing the raw Nuclear:Cytoplasmic ratios for the training data.

Click here for file

[http://www.biomedcentral.com/content/supplementary/1471-

2105-10-226-S8.zip]

\section{Additional file 9}

Test Data. Spreadsheet files containing the raw Nuclear:Cytoplasmic ratios for the test data.

Click here for file

[http://www.biomedcentral.com/content/supplementary/14712105-10-226-S9.zip]

\section{Additional file 10}

Raw counts. Counts of True Positives, False Positives and False Negatives for the training and test data.

Click here for file

[http://www.biomedcentral.com/content/supplementary/14712105-10-226-S10.doc]

\section{Acknowledgements}

Our thanks to Peter Gould who provided training data for the algorithm. This work was supported by the DTI Beacon grant "High throughput analysis of gene function in mammalian cells" and subsequently by the BBSRC SABR grant "Dynamics and function of the NF-KB signalling system" (BBF005938/BBF0056IX).

\section{References}

I. Abraham VC, Taylor DL, Haskins JR: High content screening applied to large-scale cell biology. Trends in Biotechnology 2004, 22(I): 15-22. 
2. Ainscow EK, Pilling JE, Brown NM, Orme AT, Sullivan M, Hargreaves AC, Cooke E-L, Sullivan E, Carlsson S, Andersson TB: Investigations into the liver effects of ximelagatran using high content screening of primary human hepatocyte cultures. Expert Opinion on Drug Safety 2008, 7(4):35I-365.

3. Taylor DL, Giuliano KA: Multiplexed high content screening assays create a systems cell biology approach to drug discovery. Drug Discovery Today: Technologies 2005, 2(2): 149-154.

4. Young DW, Bender A, Hoyt J, McWhinnie E, Chirn G-W, Tao CY, Tallarico JA, Labow M, Jenkins JL, Mitchison TJ, et al: Integrating high-content screening and ligand-target prediction to identify mechanism of action. Nat Chem Biol 2008, 4(I):59-68.

5. Nelson DE, Ihekwaba AEC, Elliott M, Johnson JR, Gibney CA, Foreman BE, Nelson G, See V, Horton CA, Spiller DG, et al:: Oscillations in NF-kappa B signaling control the dynamics of gene expression. Science 2004, 306(5696):704-708.

6. Gilmore TD: Introduction to NF-kappa B: players, pathways, perspectives. Oncogene 25(5I):6680-6684

7. Hayden MS, Ghosh S: Shared Principles in NF-kappa B Signaling. Cell 2008, I32(3):344-362.

8. Shen H, Nelson G, Kennedy S, Nelson D, Johnson J, Spiller D, White MR, Kell DB: Automatic tracking of biological cells and compartments using particle filters and active contours. Chemometrics and Intelligent Laboratory Systems 2006, 82(I-2):276-282.

9. Shen H, Nelson G, Nelson DE, Kennedy S, Spiller DG, Griffiths T, Paton N, Oliver SG, White MRH, Kell DB: Automated tracking of gene expression in individual cells and cell compartments. Journal of the Royal Society Interface 2006, 3(I I):787-794.

10. Jameson D, Garwood K, Garwood C, Booth T, Alper P, Oliver SG Paton NW: Data Capture in Bioinformatics: Requirements and Experiences with Pedro. BMC Bioinformatics 2008, 9:183.

11. Hancock D, Wilson M, Velarde G, Morrison N, Hayes A, Hulme H, Wood AJ, Nashar K, Kell DB, Brass A: maxdLoad2 and maxdBrowse: standards-compliant tools for microarray experimental annotation, data management and dissemination. BMC Bioinformatics 2005, 6:264.

12. Parkinson $H$, Kapushesky $M$, Shojatalab M, Abeygunawardena $N$, Coulson R, Farne A, Holloway E, Kolesnykov N, Lilja P, Lukk M, et al.: ArrayExpress - a public database of microarray experiments and gene expression profiles. Nucl Acids Res 2007 35(suppl_I):D747-750.

13. Jones P, Cote RG, Cho SY, Klie S, Martens L, Quinn AF, Thorneycroft $D$, Hermjakob H: PRIDE: new developments and new datasets. Nucl Acids Res 2008, 36(suppl_I):D878-883.

14. LSM Image Browser [http://www.zeiss.com/lsm]

15. Goldberg I, Allan C, Burel J-M, Creager D, Falconi A, Hochheiser H, Johnston J, Mellen J, Sorger P, Swedlow J: The Open Microscopy Environment (OME) Data Model and XML file: open tools for informatics and quantitative analysis in biological imaging. Genome Biology 2005, 6(5):R47.

16. Schiffmann DA, Dikovskaya D, Appleton PL, Newton IP, Creager DA, Allan C, Nathke IS, Goldberg IG: Open Microscopy Environment and FindSpots: integrating image informatics with quantitative multidimensional image analysis. Biotechniques 2006, 4I:199-209.

17. Bio-formats Library [http://loci.wisc.edu/ome/formats.html]

18. Martone ME, Tran J, Wong WW, Sargisa J, Fong L, Larson S, Lamont SP, Gupta A, Ellisman MH: The Cell Centered Database project: An update on building community resources for managing and sharing 3D imaging data. Journal of Structural Biology 2008, I6I(3):220-231.

19. Martone ME, Gupta A, Wong M, Qian XF, Sosinsky G, Ludascher B, Ellisman $\mathrm{MH}$ : A cell-centered database for electron tomographic data. Journal of Structural Biology 2002, I 38(I-2): | 45- I 55.

20. Bisque Database [http://www.bioimage.ucsb.edu/bisque]

21. Müller H, Michoux N, Bandon D, Geissbuhler A: A review of content-based image retrieval systems in medical applications clinical benefits and future directions. International Journal of Medical Informatics 2004, 73(1): I-23.

22. Moustakas J, Marias K, Dimitriadis S, Orphanoudakis SC, leee: Twolevel CBIR platform with application to brain MRI retrieval. IEEE International Conference on Multimedia and Expo (ICME): Jul 06-08 2005; Amsterdam, NETHERLANDS 2005: 1279-1282.

23. Chang YC, Antani S, Lee DJ, Gledhill K, Long LR, Christensen P: CBIR of spine $X$-ray images on inter-vertebral disc space and shape profiles. 2 I st IEEE International Symposium on Computer-Based Medical Systems: Jun 17-19 2008; Jyvaskyla, FINLAND 2008:224-229.

24. Lin C, Mak W, Hong P, Sepp K, Perritnon N: Intelligent interfaces for mining large-scale RNAi-HCS image databases. 7th IEEE International Conference on Bioinformatics and Bioengineering: Oct I 4-17 2007; Boston, MA 2007: I333-1337.

25. Brazma A, Hingamp P, Quackenbush J, Sherlock G, Spellman P, Stoeckert C, Aach J, Ansorge W, Ball CA, Causton HC, et al:: Minimum information about a microarray experiment (MIAME) - toward standards for microarray data. Nature Genetics 200I, 29(4):365-37I.

26. Taylor CF, Paton NW, Lilley KS, Binz P-A, Julian RK, Jones AR, Zhu W, Apweiler R, Aebersold R, Deutsch EW, et al:: The minimum information about a proteomics experiment (MIAPE). Nature Biotechnology 2007, 25(8):887-893.

27. Garwood KL, Taylor CF, Runte KJ, Brass A, Oliver SG, Paton NW: Pedro: a configurable data entry tool for XML. Bioinformatics 2004, 20( I 5):2463-2465.

28. Tamino - The XML Database [http://softwareag.com/tamino]

29. eXist Open Source Native XML Database [http://exist.source forge.net/]

30. Peak Finding and Measurement [http:www.mathrks.comatlabcentraleexchangloadFile.do?objectld I ctType=file]

3I. Draghici S, Tarca AL, Yu L, Ethier S, Romero R: KUTE-BASE: storing, downloading and exporting MIAME-compliant microarray experiments in minutes rather than hours. Bioinformatics 2008, 24(5):738-740.
Publish with Biomed Central and every scientist can read your work free of charge

"BioMed Central will be the most significant development for disseminating the results of biomedical research in our lifetime. "

Sir Paul Nurse, Cancer Research UK

Your research papers will be:

- available free of charge to the entire biomedical community

- peer reviewed and published immediately upon acceptance

- cited in PubMed and archived on PubMed Central

- yours - you keep the copyright 